\title{
Acute complications following intracavitary high-dose-rate brachytherapy in uterine cancer
}

\author{
Phalguni Gupta, MD', Ranen Kanti Aich, MD', Asit Ranjan Deb, MD, DNB² \\ 'Department of Radiotherapy, Nil Ratan Sirkar Medical College \& Hospital, Kolkata, ${ }^{2}$ Department of Radiotherapy, Medical College Hospital, \\ Kolkata, West Bengal, India
}

\begin{abstract}
Purpose: Almost 30\% of malignancies in women of developing countries are gynecological and brachytherapy is an integral part of management of these patients. Reports of complications (both acute and late) of high-dose-rate (HDR) intracavitary brachytherapy are sparse in world literature due to relatively small number of gynecological malignancies, particularly in advanced stage, in developed countries. High-dose-rate brachytherapy is gaining popularity in developing countries due to scientific and economic reasons. Here we are reporting our experience regarding acute complications of intracavitary brachytherapy (events occurring within 30 days of insertion needing hospitalization or death) and their causes to improve the quality of management, so that the already low incidence of acute complications can be further reduced.

Material and methods: From February 2004 to December 2012, a total of 1947 patients with uterine cancer were treated by HDR intracavitary brachytherapy in the Department of Radiotherapy, of a tertiary cancer centre of a developing country, $86 \%$ of them were cervical cancer and $14 \%$ endometrial cancer. Excluding the post-operative patients, a total of 4285 insertions were done in 1527 patients with intact uterus (eligible for analysis) and acute complications were analyzed.

Results: Out of 4285 intracavitary brachytherapy insertions in gynecological malignancy patients, only 12 mortality and 239 morbidity instances needing hospitalization were documented and most of them were in cervical carcinoma patients.

Conclusions: Our results have indicated that acute complications can be minimized by pre-treatment management of co-morbidities, decreasing the time of operative lithotomy position and bed rest, avoidance of 'conscious sedation' in selected cases etc. Routine post insertion CT scan if done in all patients in all insertions, then only, uterine perforations can be detected early and prompt management can reduce both the mortality and morbidity to a great extent.

Key words: acute complications, cervical cancer, HDR brachytherapy, intracavitary brachytherapy, uterine cancer.

\section{Purpose}

In developing countries, cervical cancer is the commonest malignancy among women, and India is no exception. In our hospital, it represents about $24 \%$ of the overall malignancies in females. Due to lack of adequate screening program combined with very low health consciousness and infrastructural deficiencies, majority of these patients present at an advanced stage, mostly in IIIB and some in IIB as per FIGO staging system. Treatment of choice in these patients is radiotherapy, which is a combination of both external beam radiotherapy (EBRT) and intracavitary brachytherapy (ICRT) of which former one is usually combined with concurrent chemotherapy. Aim of EBRT is to control the pelvic disease as well as to reduce the size of central tumor, later of which is ultimately controlled by ICRT.
Brachytherapy sources have undergone a full evolution from manual pre-loaded low-dose-rate (LDR) to remote controlled after loaded high-dose-rate (HDR). Advantages of HDR sources are manifold, e.g. elimination of radiation exposure to the attending physician and other health personnel, patient convenience, better dose optimization due to short overall treatment time, greater sparing of the rectum and bladder by temporary spacing, inter-digitations with EBRT, out-patient basis treatment delivery resulting in decreased need of hospitalization etc. [1]. In addition, most modern afterloaded sources are stepping source that allow optimization of dose distribution by varying the dwell time and positions.

The term 'acute complication' means 'events occurring within 30 days from the date of insertion of brachytherapy necessitating hospitalization or death'. Due to few number of cervical cancer patients in developed countries

Address for correspondence: Phalguni Gupta, MD, Department of Radiotherapy, Nil Ratan Sirkar Medical Received: 23.12.2013 College \& Hospital, Kolkata, West Bengal, India, A-7/9, E.K.T.P (Phase-II), Kolkata 700 107, West Bengal, 
and still fewer number in advanced stage, information in this respect in world literature is sparse.

Here, we are reporting our experience of acute complications following HDR brachytherapy in gynecological malignancies, and have tried to identify the causative factors to further reduce the already low rate of acute complications.

\section{Material and methods}

From February 2004 to December 2012, a total of 1947 patients with gynecological malignancies were treated by EBRT and ICRT. Most of them were diagnosed with cervical cancer, a minority - with endometrial cancer.

Before starting treatment, patients were evaluated thoroughly and the evaluation criteria were:

1) histologically proved carcinoma of the cervix or endometrium and completed EBRT;

2) staging as per FIGO recommendation;

3) hematological parameters were as follows: a) hemoglobin: $\geq 10 \mathrm{gm} \%$; b) platelet count: $\geq 100000 / \mathrm{mm}^{3}$; c) serum creatinine: $\leq 1.5$ times of normal value; $d$ ) bleeding and clotting times: within normal limits;

4) electrocardiogram: within normal limits;

5) post-prandial blood sugar: within normal limits;

6) X-ray chest and pulmonary function tests: within normal limits;

7) ultrasonography/CT scan of whole abdomen, particularly to note status of pre and para aortic lymph nodes;

8) MRI of pelvis, if possible;

9) informed consent.

Patients, who did not fulfill the above mentioned criteria 3-6, were first given appropriate management and then treatment for malignancy began. Patient evaluation results have been shown in Table 1.

\section{Treatment schedule}

All patients had already been treated by EBRT at a dose of $50 \mathrm{~Gy} / 25$ fractions/ 5 weeks by parallel opposed antero-posterior beams to the whole pelvis in Cobalt-60 machine (Theratron 780E, Theratronics, Canada). Bulky patients having inter-field distance $\geq 18 \mathrm{~cm}$, were treated by four field box technique. Most of the cervical cancer patients had also received concurrent chemotherapy with cisplatin $40 \mathrm{mg} / \mathrm{m}^{2}$ weekly $\times 5 \mathrm{such}$. For cervical cancer patients, an ICRT dose of $7 \mathrm{~Gy}$ at Manchester point ' $\mathrm{A}$ ' weekly for 3 insertions was recommended. In patients with intact uterus, an uterine tandem and a pair of an ovoids in the vaginal fornices were inserted. Postoperative cervical cancer patients were treated only with ovoids. In all cases, rectal separator followed by tight vaginal gauze packing was done to increase the distance between the radiotherapy sources and anterior rectal and posterior bladder walls. Post operative endometrial cancer patients received an ICRT dose of 6 Gy at a depth of $5 \mathrm{~mm}$ from the surface of the vaginal mucous membrane for 3 fractions at one week apart by vaginal cylinder. Patients with intact uterus were treated like cervical cancer patients.

All the ICRT procedures were carried out on 'Outdoor Patient Department (OPD)' basis under deep sedation. A Foley's catheter was inserted into the urinary bladder and radio-opaque solution was pushed into the bulb of the catheter to delineate the posterior bladder wall. Because of the narrow width of the intrauterine tandem, cervical dilatation was rarely required. In some patients where intrauterine tandem could not be administered because of anatomical distortion or cervical fibrosis due to disease process, only ovoids had to be used. After completion of the insertion process and vaginal packing, a rubber tube containing thick barium sulphate solution was inserted into the rectal canal, and treatment planning was done with the help of C-arm

Table 1. Pre-treatment patient characteristics $(n=1947)$

\begin{tabular}{|c|c|c|}
\hline Criteria & $\begin{array}{l}\text { Cervical cancer } \\
\quad(n=1668)\end{array}$ & $\begin{array}{c}\text { Endometrial } \\
\text { cancer } \\
(n=279) \\
\end{array}$ \\
\hline \multicolumn{3}{|l|}{ Age } \\
\hline Range & $32-69$ years & 45-74 years \\
\hline Median & 41 years & 58 years \\
\hline \multicolumn{3}{|l|}{ Operative status } \\
\hline Postoperative & $200(12 \%)$ & $220(79 \%)$ \\
\hline Inoperable & $1468(88 \%)$ & 59 (21\%) \\
\hline \multicolumn{3}{|l|}{ Stage (FIGO) } \\
\hline । & $71(4 \%)$ & $114(41 \%)$ \\
\hline$\|$ & $468(28 \%)$ & $106(38 \%)$ \\
\hline III & $1033(62 \%)$ & 45 (16\%) \\
\hline IV & $96(6 \%)$ & $14(5 \%)$ \\
\hline \multicolumn{3}{|c|}{ Karnofsky performance status } \\
\hline$\geq 90$ & $1108(66 \%)$ & $154(55 \%)$ \\
\hline $80-90$ & $341(20 \%)$ & $92(33 \%)$ \\
\hline $70-80$ & $197(12 \%)$ & $24(9 \%)$ \\
\hline $60-70$ & $22(1 \%)$ & $9(3 \%)$ \\
\hline \multicolumn{3}{|l|}{ Hemoglobin } \\
\hline$>10 \mathrm{gm} \%$ & 1301 (78\%) & $248(89 \%)$ \\
\hline$<10 \mathrm{gm} \%$ & 367 (22\%) & $31(11 \%)$ \\
\hline \multicolumn{3}{|l|}{ Serum creatinine } \\
\hline$\leq 1.5$ times of normal & $1384(83 \%)$ & $251(90 \%)$ \\
\hline$\geq 1.5$ times of normal & 284 (17\%) & $28(10 \%)$ \\
\hline \multicolumn{3}{|l|}{ Echocardiogram } \\
\hline Within normal limits & 1535 (92\%) & $234(84 \%)$ \\
\hline Outside normal limits & $133(8 \%)$ & 45 (16\%) \\
\hline \multicolumn{3}{|l|}{ Postprandial blood sugar } \\
\hline Within normal limits & 1568 (94\%) & 215 (77\%) \\
\hline Outside normal limits & $100(6 \%)$ & $64(23 \%)$ \\
\hline
\end{tabular}


visualization (Mexicon 2000, M E X-ray India Pvt. Ltd, India) and Treatment Planning System (TPS, Nucletron, an Elekta company, Elekta AB, Stockholm, Sweden) till 2010. Since 2010, C-arm was replaced by dedicated computerized tomography (CT scan, Somatom Emotion 16, Siemens Ltd, Kolkata, India). When there was a sudden loss of resistance at the time of uterine sound/tandem insertion or uterine cavity length was found to be unusually long, an uterine perforation was suspected, to be confirmed or excluded by immediate pelvic USG/CT scan, and either the procedure was postponed or continued. All patients with uterine perforation and extensive vault/vaginal lacerations were immediately hospitalized and managed accordingly. When postponed, the ICRT procedure was resumed one week after the patient was stabilized. When perforation either complete or partial has occurred in any attempt, post insertion USG/CT scan was done routinely in subsequent insertions even when perforation was not suspected. Prophylactic antibiotic was prescribed in all patients who had intrauterine manipulation or vaginal laceration and oral analgesics to all patients.

After completion of treatment, patients were followed up at two weeks interval for two months and then at six weeks interval for the next six months. Follow up attendance was not very regular. Non compliance to follow up was almost $3 \%$ at one month, which gradually increased to $11 \%$ at six months. After that period, follow up interval was gradually increased and follow up continued till death with late complications documented, which will be discussed at a later date.

\section{Results}

Patient characteristics is presented in Table 1. Cervical cancer patients had a median age of 41 years (32-69 years) and only $12 \%$ of them were postoperative. Endometrial cancer patients had a median age of 58 years (45-74) and 79\% of them were postoperative. Regarding staging, stage III was the most common (62\%) in cervical cancer patients followed by stage II ( $28 \%)$, whereas, most of the endometrial cancer patients were of stage I ( $41 \%)$ and II (38\%). Most of the patients of both the groups had Karnofsky Performance Status $\geq 90$.

Pre-ICRT evaluation results have also been shown in Table 1. In comparison to endometrial cancer, more cervical cancer patients had hemoglobin level < $10 \mathrm{gm} \%(22 \%$ vs. $11 \%)$ and deranged kidney function (17\% vs. $10 \%)$. These patients received blood transfusion and management from the nephrologists before starting ICRT. Similarly, abnormal electrocardiogram and raised blood sugar were more common in endometrial cancer patients $(16 \%$ vs. $0.8 \%$ and $23 \%$ vs. $0.6 \%$ ), and were treated accordingly.

Though three ICRT insertions were planned in all patients, the prescribed treatment could not be completed in all of them. Treatment with both tandem and ovoids was attempted in 1468 cervical and 59 endometrial (with intact uterus) cancer patients. Tandem could not be inserted in 54 (3.67\%) cervical and 7 (11.86\%) endometrial cancer patients. Nine cervical cancer patients did not take ICRT beyond $1^{\text {st }}$ insertion. One of them died due to acute complications, following complete uterine perforation, and the rest did not turn up for second insertion for unknown reason. In addition, 8 more cervical cancer patients had complete and 21 partial perforation. All patient recovered. Similarly, 91 cervical cancer patients did not proceed beyond $2^{\text {nd }}$ insertion. Thirteen of them had complete uterine perforation of whom 4 died, rest recovered, 16 partial uterine perforation, vault laceration in 2, deep vein thrombosis in 2 , fever in 2 , and the rest did not turn up for unknown reason. In endometrial cancer patients, 4 of them completed only two insertions; 1 of them died following complete perforation and the other $3 \mathrm{did}$ not turn up 1 of whom had deep vein thrombosis. Eleven cervical and 3 endometrial cancer patients detected with complete uterine perforation after $3^{\text {rd }}$ insertion of whom, 5 cervical and 1 endometrial cancer patient died, rest recovered. Similarly, partial uterine perforation was detected in 29 cervical and 3 endometrial cancer patients after $3^{\text {rd }}$ insertion and all recovered. The result of insertion attempts has been shown in Table 2 .

The median insertion time was about 35 minutes in our patients. Once the tandem and ovoids were in position, rectal separator applied and vaginal packing done, the patients were placed in supine position during the imaging, calculation, and irradiation procedure. Another 40-50 minutes were required for imaging and calculation followed by 8-35 minutes for dose delivery depending upon the source output. When uterine perforation occurred in any insertion attempt, routine post insertion USG/CT were done in subsequent attempts, even when perforation was not suspected.

In our institution, in the above mentioned 9 years period, only 239 acute events had occurred consisting of 12 mortalities and 227 morbidities. The events have been tabulated in Table 3. Two point thirteen percent $(2.13 \%)$ patients were detected to have complete uterine perforation by USG and the figure rose to $4.12 \%$ in post-CT era. But if we consider the total number of insertions done, then the percentages become $0.73 \%$ and $1.38 \%$ in pre- and post-CT era. These percentages were calculated based on the number of patients in whom tandem insertion was attempted. During this period, 12 patients died due to uterine perforation, 11 in the pre-CT, and 1 in the post-CT scan era. In the pre-CT period, USG were done only in the suspected cases of perforation, and in 10 of the patients perforation was either not suspected or not detected. They came back after 3-4 days with features of peritonitis; perforation detected retrospectively and could not be salvaged. One patient in the pre-CT era and 1 who died in the post-CT era, perforation was detected during tandem insertion, admitted in the hospital, treated conservatively, but the gut injury remained undetected and died of peritonitis. Rest 25 patients, in whom complete uterine perforation was detected during ICRT procedure, were kept admitted, treated conservatively, and recovered. Most of the partial uterine perforations were detected in the post-CT era and were managed conservatively with antibiotics, fluid, and blood transfusion, when needed. Vault or vaginal lacerations were treated with tight gauge packing under anesthesia, sedation, antibiotics, 
Table 2. Results of planned intracavitary brachytherapy (ICRT) insertions ( $n=1527)$. Only tandem and ovoids patients

\begin{tabular}{lcccc} 
Patients (pts) & \multicolumn{2}{c}{ Cervix $(n=1468)$} & \multicolumn{2}{c}{ Endometrium $(n=59)$} \\
\cline { 2 - 5 } & Pre-CT scan era & Post-CT scan era & Pre-CT scan era & Post-CT scan era \\
\hline No. of pts & 1176 & 292 & 42 & 17 \\
\hline No. of pts completed 3 insertions & 1055 & 259 & 33 & 15 \\
\hline No. of pts completed 2 insertions only & 72 & 4 & 0 & 1 \\
\hline No. of pts completed 1 insertion only & 5 & 10 & 6 & 1 \\
\hline No. of pts in whom tandem could not be inserted & 44 & 819 & 105 & 47
\end{tabular}

Table 3. Description of acute events $(n=239)$. Only in tandem and ovoids patients; total 4285 insertions

\begin{tabular}{|c|c|c|c|c|c|c|}
\hline \multirow[t]{2}{*}{ Acute events } & \multicolumn{2}{|c|}{ No (\%) } & \multirow{2}{*}{$\begin{array}{l}\text { Treatment done, } \\
\qquad n=\end{array}$} & \multicolumn{2}{|c|}{ Results } & \multirow{2}{*}{$\begin{array}{l}\text { Cause of death, } \\
\qquad n=\end{array}$} \\
\hline & Pre-CT era & Post-CT era & & Pre-CT era & Post-CT era & \\
\hline $\begin{array}{l}\text { Complete uterine } \\
\text { perforation }\end{array}$ & $25(0.73)$ & $12(1.38)$ & $\begin{array}{c}C M, B T \text { in } 26, \\
A H \text { in } 9, \\
A H+G R \text { in } 6\end{array}$ & $\begin{array}{l}11 \text { died, } \\
14 \text { recovered }\end{array}$ & $\begin{array}{l}1 \text { died, } \\
11 \text { recovered }\end{array}$ & $\begin{array}{l}\text { Gut injury leading } \\
\text { to sepsis: } 8 \\
\text { Intra-peritoneal } \\
\text { bleeding: } 4\end{array}$ \\
\hline $\begin{array}{l}\text { Partial uterine } \\
\text { perforation }\end{array}$ & $13(0.38)$ & $56(6.46)$ & CM & \multicolumn{2}{|c|}{ All recovered } & \\
\hline Vault laceration & \multicolumn{2}{|c|}{$6(0.14)$} & Vault suturing in 2, CM & \multicolumn{2}{|c|}{ All recovered } & \\
\hline Vaginal laceration & \multicolumn{2}{|c|}{$5(0.11)$} & CM & \multicolumn{2}{|c|}{ All recovered } & \\
\hline $\begin{array}{l}\text { Deep vein } \\
\text { thrombosis }\end{array}$ & \multicolumn{2}{|c|}{$16(0.37)$} & CM & \multicolumn{2}{|c|}{ All recovered } & \\
\hline Fever & \multicolumn{2}{|c|}{106 (2.47) } & CM & \multicolumn{2}{|c|}{ All recovered } & \\
\hline
\end{tabular}

and blood transfusion, if required, and only 2 of them required suturing. Deep vein thrombosis and fever were treated conservatively and all recovered.

\section{Discussion}

High-dose-rate brachytherapy is gaining popularity even in developing countries. Apart from scientific reasons, cost effectiveness is a compelling criterion of its popularity. Due to vast number of patients suffering from gynecological malignancies, particularly from carcinoma cervix of advanced stage combined with relatively few number of available brachytherapy units, a large number of patients are to be treated per day. Time has come when we will be cautious about the acute and late effects of HDR brachytherapy. It is dangerous to assume that HDR brachytherapy is associated with less acute complications than LDR brachytherapy. As patients are treated on OPD basis, they can't be observed for a certain length of time post ICRT and acute complications may progress to a certain extent before diagnosis, leading to increased morbidity and mortality. Acute complications related to LDR brachytherapy are gynecological malignancies and have been described in some literature. Corn et al. [2] analyzed acute complications in 143 insertions (100 patients) of tandem/colpostat in cervical cancer patients in 20 of whom insertions were done under ultrasound guidance. Complications occurred in 7 of 143 placements (5\%). These included uterine perforations (4), vaginal lacerations (2), and 1 instance of bladder perforation. Postoperative complications (e.g., fever, bowel obstruction, exacerbation of chronic obstructive pulmonary disease, cardiac complication) occurred in 54 out of 143 placements. A multivariate analysis showed that underlying COPD predisposed to postoperative complications during the first implant, and that age over 60 years independently predicted for complications during any implant. They concluded that intraoperative complications are rare and use of ultrasonography allows safe tandem insertion. Incidence of uterine perforation was $2.79 \%$ in Corn's report, and $2.47 \%$ of insertions in our series. Lanciano et al. [3] evaluated the incidence and severity of perioperative morbidity with standard tandem and ovoid insertions in gynecological malignancies. Ninety-one cervical and 4 endometrial cancer patients were chosen for this study. Intra-operative complications developed in 3\% of insertions including perforation and vaginal laceration. Five percent of the insertions had to be removed emergently secondary to pre- 
sumed sepsis, exacerbation of chronic obstructive pulmonary disease, hypotension, change in mental status, and myocardial infarction/congestive heart failure.

Sarkaria et al. [4] assessed the 30-day morbidity and mortality rates for patients with an intact uterus undergoing HDR brachytherapy, and the overall acute events occurred in $9.5 \%$ of patients. Their overall morbidity and mortality rates were similar to LDR brachytherapy, but higher than other HDR reports and age, turned out to be the most significant predictive factor. Mean procedural time exceeding 160 minutes emerged to be very significant. The overall acute events occurred in $5.57 \%$ of our insertions, which is much lower than reported by Sarkaria et al. Dusenbery et al. [5] reported 30 days morbidity and mortality rate of $6.4 \%$ and $1.5 \%$, respectively, following HDR brachytherapy. Their series included both cervical and postoperative endometrial carcinoma patients. Chao et al. [6] reported $4.2 \%$ and $2.1 \%$ of morbidity and mortality rates, respectively, in gynecological malignancies, and his series involved inoperable endometrial carcinoma patients.

Our morbidity rate was a bit higher $(5.29 \%)$, but mortality rate $(0.28 \%)$ was much lower than Chao et al. However, the largest trial to date has been published by Knocke et al. [7], in which 280 medically inoperable endometrial cancer patients were treated by HDR brachytherapy and experienced no acute complication.

Petereit et al. [8] reported 30 day morbidity and mortality for cervical and medically inoperable endometrial carcinoma patients as $5.5 \%$ and $1.6 \%$, and $9.8 \%$ and $7.3 \%$, respectively, with HDR brachytherapy. They identified certain risk factors associated with acute complications e.g. age by decades, performance status, cardiac problems, American Society of Anesthesiologists (ASA) physical class system, positive medical history etc. They predicted age as the main factor influencing morbidity and mortality. A relative risk of 2.0 per decade implies that an 80 year old woman has an estimated 16 fold higher risk than a year old women. All other risk factors were correlated with age. Dusenbery et al. [5] and Lanciano et al. [3] have also stressed on age, cardiac problems, type of anesthesia used etc, as factors influencing acute complications.

In our patients, rate of acute complications were significantly low and we have tried to analyze the factors responsible for this low rate of acute complications in comparison to published reports. As age has turned out to be the most important factor influencing acute complication, it is quite clear why acute complications were less in our patients. Median age of our cervical and endometrial cancer patients was 41 years (range: $32-69$ years) and 58 years (range: 45-74 years), respectively, whereas the mean age of Petereit's [8] patients was 50 years (range: $23-88$ years) and 63 years (range: $37-81$ years), respectively.

Deep vein thromboses were identified as an important cause of acute complications. Petereit et al. [8] reported an incidence of $7.3 \%$ and $1.8 \%$ respectively, in endometrial cancer and overall patients. But, incidences of deep vein thrombosis were $1.2 \%$ and $1.0 \%$, respectively, in Dusenbery and Corn series [2,5]. Rotte observed no deep vein thrombosis in his patients [9]. Similarly, we have observed only $16(0.37 \%)$ incidence of clinically significant deep vein thrombosis in our patients. The cause of this low incidence of deep vein thrombosis probably lies in the racial variation, age, procedural time, and pre-treatment management. Deep vein thrombosis is less common in Asian than Western population. The median procedural times in Petereit series were 133 minutes and 160 minutes, respectively, for cervical and endometrial cancer patients, and the patients were kept in dorsal lithotomy position during this whole period of procedure. The median procedural time was about 100 minutes in our patients. This low procedural time is probably responsible for the low incidence of deep vein thrombosis in our patients.

Accurate positioning of the intra-cavitary applicators are critical for delivering appropriate doses of irradiation to the target volume while keeping the doses to the surrounding organs at risk below their tolerance limits. Consequently, unrecognized uterine perforation may lead to under dosage of the target volume, compromising local control probability [10]. In addition, the perforating applicator may come in direct contact or in vicinity of the organs at risk, leading to their exposure to excessive doses, resulting in acute and long-term gastrointestinal, and genitourinary complications. Incidence of uterine perforation, particularly partial perforation during tandem insertion is probably underappreciated in our series. Complete perforation was $0.73 \%$ in pre-CT scan era, but $1.38 \%$ in post-CT scan period. As USG were done only in suspected cases of perforation, it may easily be assumed that a lot of sub-clinical perforations have been missed. Similarly, incidence of partial perforation rose from $0.38 \%$ to $6.46 \%$ with the introduction of routine CT scan. It appeared that USG is a very unreliable tool to detect partial perforation. Petereit et al. documented a $2 \%$ incidence of uterine perforation in their patients, but many of these perforations were partial, dissected the anterior or posterior uterine wall rather than puncturing the uterine serosa. Segedin et al. [11] tried to determine the incidence of uterine perforation by 3D imaging with the applicators in position, and found the incidence to be $3 \%$ only and all cases were managed conservatively. Uterine perforation occurs more frequently in patients with anatomical distortions of the cervix and/or cervical stenosis due to advanced disease, post irradiation fibrosis and previous cone biopsy. Patients over 60 years are particularly predisposed to uterine perforation, probably due to vaginal atrophy and anatomical distortion of the cervix. Other predisposing factors include retro-verted/retro-flexed or extremely ante-verted/ante-flexed uterus [12]. In our institution, ICRT was started one week after completion of EBRT and therefore chances of anatomical distortion of cervix or post radiation fibrosis were much less and cone biopsy was not done in any of them.

Another cause of mortality is sedation. Most series had used 'conscious sedation' - the goal of which was to relieve anxiety and pain and to promote amnesia [13]. To achieve these goals, a benzodiazepine is typically combined with a narcotic analgesic [14]. Petereit series had observed almost $10 \%$ mortality in the inoperable corpus 
group. As we have used deep sedation (combining inj. diazepam and pentazocin) in our patients, no mortality in this respect has been noticed.

\section{Conclusions}

High-dose-rate brachytherapy is gaining popularity in developing countries where it is mostly needed as almost $30 \%$ of malignancies of women of these countries are gynecological. But probably none has published their reports of acute complications of HDR brachytherapy in gynecological malignancies, particularly in OPD settings. Our study showed that the acute complications of intracavitary brachytherapy can be minimized by pre-treatment management of associated comorbidities, decreasing the duration of operative lithotomy position, routine use of 3D imaging in all insertions etc.

\section{Disclosure}

Authors report no conflict of interest.

\section{References}

1. Sarkaria JN, Petereit DG, Stitt JA et al. A comparison of the efficacy and complication rates of low dose-rate versus high dose-rate brachytherapy in the treatment of uterine cervical carcinoma. Int J Radiat Oncol Biol Phys 1994; 30: 75-82.

2. Corn BW, Barry D. Shaktman BD et al. Intra and perioperative complications associated with tandem and colpostat application for cervix cancer. Gynecol Oncol 1997; 64: 224-229.

3. Lanciano R, Corn B, Martin E et al. Perioperative morbidity of intracavitary gynecologic brachytherapy. Int J Radiat Oncol Biol Phys 1994; 5: 969-974.

4. Sarkaria JN, Petereit DG, Kinsella TJ et al. An analysis of acute complications and perioperative morbidity from high dose rate brachytherapy in the treatment of gynecological malignancies. Int J Radiat Oncol Biol Phys 1995; 32 (Suppl. 1): 224.

5. Dusenbery KE, Carson LF, Potish RA. Perioperative morbidity and mortality of gynecologic brachytherapy. Presented at the $72^{\text {nd }}$ Annual Meeting of the American Radium Society, Scottsdale, Arizona, April 21-25, 1990.

6. Chao CKS, Grigsby PW, Perez CA et al. Brachytherapy related complications for medically inoperable stage I endometrial carcinoma. Int J Radiat Oncol Biol Phys 1995; 1: 37-42.

7. Knocke TH, Kucera H, Weidinger B et al. Primary treatment of endometrial carcinoma with high dose rate brachytherapy: Results of 12 years of experience with 280 patients. Int J Radiat Oncol Biol Phys 1997; 37: 359-365.

8. Petereit DG, Sarcaria JN, Chappell RJ. Perioperative morbidity and mortality of high dose rate gynecologic brachytherapy. Int J Radiat Oncol Biol Phys 1998; 42: 1025-1031.

9. Rotte K. Technique and results of HDR after loading in cancer of endometrium. In: Brachytherapy HDR and LDR. Martinez A, Orton CG, Mould RF (eds.). Nucleotron, Columbia 1990; 68-79.

10. Corn BW, Hanlon AL, Pajak TF et al. Technically accurate intracavitary insertions improve pelvic control among patients with locally advanced carcinoma of the uterine cervix. Gynecol Oncol 1994; 53: 294-300.

11. Segedin B, Gugic J, Petric P. Uterine perforation - 5-year experience in 3-D image guided gynaecological brachytherapy at Institute of Oncology Ljubljana. Radiol Oncol 2013; 47: 154-160.

12. Barnes EA, Thomas G, Ackerman I et al. Prospective comparison of clinical and computed tomography assessment in detecting uterine perforation with intracavitary brachytherapy for carcinoma of the cervix. Int J Gynecol Cancer 2007; 17: 821-826.

13. Brandt KA, Petereit DG, Sarkaria JN et al. Conscious sedation in outpatient high dose rate gynecologic brachytherapy. Proceedings from $1^{\text {st }}$ Annual Multidisciplinary Radiation Oncology Conference. Philadelphia, 1996; 206.

14. White PF. The role of midazolam in outpatient anesthesia. Anesth Rev 1985; 12: 55-60. 